\title{
Revisión bibliográfica de la marca corporativa: hacia una definición y gestión integradoras
}

\section{Review of the literature on corporate brands: towards an integrative definition and management}

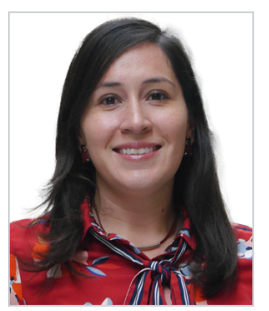

Tatiana Pereira-Villazón. Realiza su doctorado en la Universidad de Navarra en el Departamento Marketing y Empresas de Comunicación. Tiene un Máster en Comunicación Corporativa por la Universidad de Barcelona. Sus intereses de investigación se relacionan con la creación y gestión de marcas corporativas, con especial interés en la industria audiovisual, y los principales cambios en la gestión de la marca de medios.

Universidad de Navarra, España

tpereira@alumni.unav.es

ORCID: 0000-0003-4238-5300

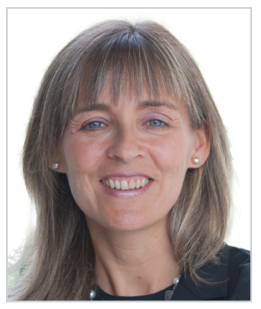

Idoia Portilla. Profesora de la Facultad de Comunicación de la Universidad de Navarra, es doctora en CC. Económicas y Empresariales por esta universidad. Sus principales áreas de interés son la medición de audiencias, ética y metodología en investigación de mercados, y los efectos de los nuevos medios en los tradicionales. Desde 2013, preside el Comité de Ética de la Asociación Española de Estudios de Mercado, Marketing y Opinión (AEDEMO). Universidad de Navarra, España iportilla@unav.es

ORCID: 0000-0002-2504-868X

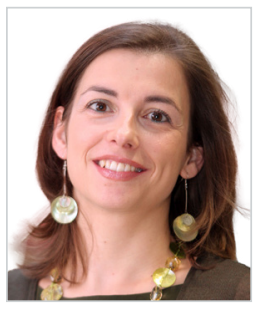

Natalia Rodríguez-Salcedo. Profesora Contratado Doctor, subdirectora del Departamento de Marketing y Empresas de Comunicación y subdirectora del Máster en Reputación Corporativa de la Facultad de Comunicación de la Universidad de Navarra. Posee una doble licenciatura en Periodismo y en Publicidad y Relaciones Públicas. Terminó su tesis doctoral en Comunicación Pública en 2004. En la actualidad, imparte clases de Introducción a las Relaciones Públicas, Comunicación Corporativa y Gestión de Asuntos Públicos en la Facultad de Comunicación de la Universidad de Navarra, donde da clases desde 2001. Coordina las menciones en Comunicación Institucional y Comunicación Corporativa para los alumnos de Periodismo y Marketing, respectivamente. Universidad de Navarra, España

nrodriguez@unav.es

ORCID: 0000-0003-0968-0447

Cómo citar este artículo:

Pereira-Villazón, T.; Portilla, I. y Rodríguez-Salcedo, N. (2021). Revisión bibliográfica de la marca corporativa: hacia una definición y gestión integradoras. Doxa Comunicación, 32, pp. 159-185. 
Recibido: 15/01/2020 - Aceptado: 08/02/2021 - En edición: 19/04/2021 - Publicado: 14/06/2021

\section{Resumen:}

La marca corporativa es un activo intangible y estratégico de gran valor que se vincula a conceptos como identidad e imagen. Esto le ha supuesto la atención de diversas disciplinas, no solo de la comunicación. El objetivo de este trabajo es identificar dichas disciplinas, los autores destacados, ofrecer una definición integradora de la marca corporativa, identificar conceptos relacionados y el área a la que se asigna su gestión. Se trata de arrojar luz sobre la importancia de una adecuada gestión de la marca corporativa. Para ello, se ha realizado una revisión bibliográfica sistematizada de los trabajos académicos publicados durante el periodo 20162018. Sobre ellos se aplica un análisis de contenido cuantitativo y cualitativo. Tras la revisión de 112 publicaciones académicas en 66 revistas, se observa la escasa aportación del área de la comunicación en contraste con la gestión y el marketing, de donde proceden también los autores más citados. Respecto a la gestión de la marca corporativa, destaca el papel de la alta dirección y el área de marketing. Sin embargo, la variedad temática de las aportaciones y el valor estratégico de la marca corporativa sugieren la necesidad de aportar un enfoque multidisciplinar en la gestión y estudio de la marca corporativa.

Palabras clave:

Marca corporativa; comunicación; marketing; análisis de contenido.

\section{Introducción}

Desde su introducción en la década de los 90, el estudio del concepto de la marca corporativa ha despertado el interés de investigadores de diversas disciplinas como el marketing, la gestión estratégica (Balmer \& Gray, 2003; Balmer et al., 2017; He \& Balmer, 2006; Schultz \& de Chernatony, 2002) y la comunicación corporativa (Balmer, 2008a). Este interés ha motivado un importante avance en el desarrollo conceptual y en la investigación empírica del término "marca corporativa", asociado en muchos casos a otros como identidad corporativa, imagen corporativa y reputación corporativa (Abratt \& Kleyn, 2012), misión y filosofía corporativa, personalidad corporativa o identificación visual (Balmer, 1995).

Algunos de estos términos, como identidad o reputación, corresponden a la disciplina de comunicación corporativa y llevan a pensar que su gestión es una cuestión que le compete (Balmer, 2008b; Biraghi \& Gambetti, 2015). Sin embargo, marca o imagen son conceptos más vinculados al marketing, abordando su gestión como parte del mix del marketing corporativo (Balmer, 2008b; Sujchaphong, Nguyen, \& Melewar, 2017). Más aún, la gestión de la marca corporativa puede considerarse un elemento crucial para cualquier organización (Balmer, 2001a, 2001b; Ind, 1997; Jo Hatch \& Schultz, 
2003a; Schultz \& de Chernatony, 2002) y un activo estratégico clave para la diferenciación de los competidores actuales y futuros (Balmer \& Greyser, 2006), lo que sugiere la necesidad de una gestión desde la alta dirección.

Ante este contexto, el primer paso es revisar qué funciones se otorgan a la comunicación corporativa y su posible vinculación con la marca corporativa y otros conceptos que se han vinculado a ella. Tras el repaso de dichos conceptos, se plantean varias preguntas sobre las disciplinas que están estudiando la marca corporativa y hablan de su gestión.

Para responder a las cuestiones que se plantean tras el marco teórico, se aplica un análisis de contenido sobre artículos recientes centrados en la "marca corporativa". Sus resultados permitirán determinar qué disciplinas y autores destacan en su estudio, y el papel de la comunicación. También se analiza el área de gestión donde se encaja, de modo que puedan realizarse recomendaciones para su estudio.

\section{Marco teórico}

Las funciones de la comunicación corporativa se fueron afianzando a medida que los stakeholders, tanto internos como externos, comenzaron a demandar más información de las organizaciones (Cornelissen, 2008). La comunicación corporativa adquiere en ese modo un enfoque integral para ejecutar la comunicación de la organización (van Riel \& Fombrun, 2008) con el fin de conectar e influir en todos los stakeholders.

Las organizaciones buscaban proyectar una imagen positiva que, a menudo, ha sido etiquetada como identidad corporativa o marca corporativa, centrada en conectar y responder a las necesidades de estos públicos con el fin de construir, mantener y proteger la reputación (Cornelissen, 2008). En este sentido, la responsabilidad central del área de comunicación corporativa exige "desarrollar iniciativas con el fin de minimizar las discrepancias no funcionales entre la identidad deseada y la imagen deseada” (van Riel, 1995: 22), teniendo en cuenta la interacción de la triada de políticas de comunicación de la empresa: estrategia-imagen-identidad.

Según van Riel \& Fombrun (2008), la comunicación puede ser capaz de influir en la percepción de los participantes y observadores de la organización, "afectando así, a la imagen, la marca y reputación de la organización" (p. 13). En consecuencia, identidad, imagen y reputación se convierten en conceptos centrales dentro de la comunicación corporativa (Podnar, 2015).

Al mismo tiempo, la literatura relacionada con estos conceptos forma parte de la génesis de la marca corporativa (Balmer \& Gray, 2003). La comunicación se considera en estos casos como enlace conceptual entre los activos que forman parte de la marca corporativa (Balmer \& Greyser, 2006; Biraghi \& Gambetti, 2015; Harris \& de Chernatony, 2001; Jo Hatch \& Schultz, 2001).

Ante estas observaciones, parece lógico encajar el estudio y la gestión de la marca corporativa dentro del área de la comunicación corporativa. Sin embargo, antes de pasar a comprobar si la literatura sustenta esta visión y la relevancia de este enfoque, conviene repasar cómo se ha desarrollado el concepto de marca corporativa y otros relacionados con él. 


\subsection{De la Identidad corporativa a la marca corporativa}

La identidad corporativa ha sido un concepto que, desde sus orígenes, ha destacado por su carácter estratégico y su interconexión con la imagen (Costa, 2001) y la estrategia de la organización. Puede relacionarse con la función "implícita de la marca”, a partir de la cual se identificaban los productos y facilitaba la diferenciación, que se materializaba en la identidad corporativa (Costa, 2001: 223).

Como apunta Villafañe (1998) la identidad corporativa constituye un conjunto de rasgos y atributos que definen su esencia, siendo algunos visibles y otros no (Villafañe, 1998). Dichos rasgos convergen en tres ejes: la historia de la organización, el proyecto empresarial y la cultura corporativa (Villafañe, 2012; Villafañe, 1999). Por tanto, es más que una representación iconográfica (Costa, 2001). Representa la personalidad de la organización, a través de la que se diferencia.

La identidad corporativa también se define como "la manifestación actual de la realidad de la compañía transmitida a través del nombre, logo, lema, marcas, productos, servicios, inmuebles, papelería corporativa, uniformes y todo tipo de elementos de evidencia tangibles creados por la organización y comunicados a todos sus públicos" (Argenti, 2013:72). La complejidad del concepto evidencia su relación con la comunicación de la empresa a un nivel fundamental. Representa la síntesis de la identidad de la organización (Villafañe, 1999), su esencia, que se expresa a través de esta identidad de diferentes maneras (Balmer, 1995), influyendo así en la imagen (González-Oñate, 2008).

Para autores como Argenti \& Druckenmiller (2004: 369), la imagen es un "un reflejo de la identidad de la organización y de su marca corporativa”. Los consumidores y otros stakeholders toman decisiones basándose más en la percepción o imagen que tengan sobre una organización que en sus productos o servicios (Argenti, 2013). Por ello, se considera que la imagen forma parte de la mezcla de atributos únicos e intransferibles de cada organización y que contribuyen a desarrollar ventajas competitivas (Balmer \& Soenen, 1997; Podnar, 2015).

Como resultado, la comprensión del término imagen puede variar según los tipos: “imagen gráfica, imagen visual, imagen material, imagen mental, imagen de empresa, imagen de marca, imagen corporativa e imagen global” (Capriotti, 2013; Costa, 1994). Sin embargo, a efectos de gestión de la comunicación en las organizaciones (Capriotti, 2013) es importante determinar la diferencia entre dos tipos de imagen: la imagen de marca y la imagen corporativa.

Se entiende que la imagen de marca representa un conjunto de asociaciones (Aaker, 1991) y/o percepciones que los consumidores tienen sobre una marca o producto (Malmelin \& Moisander, 2014). Puede "identificarse en mayor o menor grado con la imagen de la empresa" (Capriotti, 2013: 28). No es de extrañar, por tanto, que la imagen de marca haya constituido tradicionalmente un término vinculado al marketing y no a la comunicación.

Sin embargo, si hablamos de imagen corporativa, estamos ante la "integración en la mente de sus públicos de todos los inputs emitidos por una empresa en su relación con ellos” (Villafañe, 1999: 30). En consecuencia, esta imagen corporativa es la percepción de la identidad global de una organización. Todas las señales y elementos que constituyen la empresa y que se comunican al exterior, voluntaria o involuntariamente, influyen en la construcción de la imagen que tendrán los stakeholders de dicha empresa u organización. En esta línea, Einwiller \&Will (2002) consideran que la imagen repercutirá 
en la reputación corporativa para posteriormente formar parte de un "proceso sistemáticamente planificado e implementado que se considerará marca corporativa" (p. 101).

Inspirándose en la literatura sobre identidad e imagen corporativa, podemos fijar el nacimiento del estudio de la marca corporativa en la segunda mitad de la década de 1990 (Balmer, 2010; Balmer \& Wang, 2016; Biraghi \& Gambetti, 2015). Diversos autores coinciden en señalar el artículo de Balmer (1995) "Corporate branding and connoisseurship" como el primero en referirse y mencionar la importancia de la marca corporativa (Mohan, Voss, Jiménez, \& Gammoh, 2018; Schmidt \& Redler, 2018; Schroeder, 2017; Sevel, Abratt, \& Kleyn, 2018).

Balmer (1995) definió la marca corporativa como una identidad institucional que puede ser muy significativa para los diferentes stakeholders. Jo Hatch \& Schultz (2001) la alinean con el origen y las prácticas cotidianas de la organización (la cultura), dónde aspira a ir (la visión estratégica), su imagen e identidad. Knox \& Bickerton (2003:103) la consideran "la expresión visual, verbal y de comportamiento del modelo de negocio único de una organización”. Es una representación que une y representa el conjunto de productos, servicios, unidades y negocios bajo un nombre común (Podnar, 2015).

La marca corporativa se concibe así como un activo intangible que busca representar a toda la organización (Balmer, Powell, Kernstock, \& Brexendorf, 2017). Es la cara de la organización (Balmer \& Gray, 2003), aunque una cara dinámica, que expresa unos valores referenciales para los públicos internos y externos (Villagra, López, \& Monfort, 2015).

En un intento por aunar las propuestas de diversos autores y, a efectos de esta investigación, se entenderá la marca corporativa como:

- una entidad viva y dinámica (Villagra, López, \& Monfort, 2015);

- que difiere de las marcas de productos en términos de alcance y gestión (Balmer, 1995, 2001a; Balmer \& Gray, 2003);

- a través de la cual se logra el reconocimiento y una ventaja competitiva duradera, permitiendo que la compañía sea reconocible para todos los públicos (Podnar, 2015);

- que debe estar alineada con el origen y las prácticas cotidianas de la organización (cultura organizacional) y a dónde aspira a llegar (visión estratégica);

- y que refleja cómo es percibida la organización externamente (imágenes), de acuerdo con quién es verdaderamente (identidad) la organización (Jo Hatch \& Schultz, 2001; Jo Hatch \& Schultz, 2003a).

Ante esta conceptualización, cabe preguntarse de nuevo por el papel del área de comunicación corporativa en el estudio y gestión de la marca corporativa. Dada su relevancia estratégica, parece lógico pensar que se haya abordado también desde otras . Además, aunque ya se ha visto su relación con la identidad o la imagen, se precisa conocer mejor esta relación, así como qué otros conceptos -y de qué áreas- se vinculan a la marca corporativa. Finalmente, pero no menos importante, debemos conocer cómo se plantea desde la teoría la gestión de este activo, las áreas involucradas y, consecuentemente, los ámbitos académicos para contribuir a la creación de valor en la gestión diaria en las organizaciones. 


\section{Preguntas de investigación y metodología}

El marco teórico nos lleva a formularnos cuatro preguntas importantes:

1. ¿Qué disciplinas han contribuido más al estudio de la marca corporativa y qué posición ocupa la comunicación entre ellas?

2. ¿Qué autores destacan en el estudio de la marca corporativa y en qué disciplinas están ubicados?

3. ¿Qué otros conceptos, además de imagen e identidad, se vinculan a la marca corporativa y cómo se relacionan con ella?

4. ¿Desde qué áreas se recomienda gestionar la marca corporativa?

Para responder a estas preguntas se aplicará un análisis de contenido cuantitativo y cualitativo. El universo de estudio lo configurarán artículos académicos especializados que incluyen el término "marca corporativa", localizados a partir de la revisión sistematizada de las publicaciones.

Para la selección de casos se aplica un proceso sistemático (Codina, 2018) que permite identificar de manera objetiva y sintética los artículos que desarrollan el objeto de estudio (Wimmer \& Dominick, 2011). Una vez identificados los trabajos, se realiza un análisis de contenido cualitativo y cuantitativo. El primero implica la lectura completa de los artículos para conocer los autores citados, responder a la pregunta sobre los conceptos vinculados y cómo, así como localizar las áreas de gestión que son citadas en los trabajos. Además, se aplica un análisis cuantitativo a partir de las bases de datos de las revistas, de los autores citados y de las temáticas tratadas, que permitirá obtener recuentos y saber cuáles destacan. Para la localización de los trabajos se emplean las bases de datos de Scopus, Complementary Index y BASE, entre otras (Ver anexo 1$)^{1}$. Sus funcionalidades permiten, entre otros aspectos, limitar las búsquedas por términos, idiomas, disciplinas o áreas geográficas.

La búsqueda se limitó a trabajos en inglés y castellano que incluyesen la expresión "corporate brand" o "marca corporativa". No se incluyeron los trabajos que contenían estas palabras por separado. De este modo, se buscaba evitar incluir trabajos cuyo objetivo era el estudio de la marca por sí sola o de temas corporativos, pero no de la "marca corporativa".

La búsqueda no se limitó por disciplinas y/o materias, de modo que fuera posible conocer cuáles son las que trabajan el tema de marca corporativa y responder a las preguntas de investigación. Tampoco se limitó la búsqueda por área geográfica.

Una primera búsqueda de trabajos sin limitación de fecha mostró la existencia de un gran número de casos, con un total de 13.557 artículos (ver figura 1). Dado que es precisa la lectura de los trabajos, se decidió limitar la búsqueda a los trabajos publicados de 2016 a 2018, tal y como se recomienda en este tipo de situaciones (Machi y McEvoy, 2009).

Aunque el concepto objeto de estudio, el de la marca corporativa, se viene estudiando desde los 90, la limitación a años recientes no impide alcanzar una visión amplia sobre el tema, puesto que las publicaciones citan trabajos previos. Dado

1 El acceso a las citadas bases de datos se ha desarrollado a través de la herramienta Unika, disponible en la Biblioteca de la Universidad de Navarra. 
que el trabajo de campo se realiza en el 2020, no se incluye el año 2019 para evitar problemas con el embargo de revistas, que impide el acceso a los trabajos.

Tras limitar la búsqueda al periodo de 2016 a 2018, la muestra se redujo a 541 trabajos. A continuación, se eliminaron las publicaciones duplicadas por estar presentes en más de una base de datos, excluyendo 323 casos, dejando un total de 218 publicaciones.

Figura 1

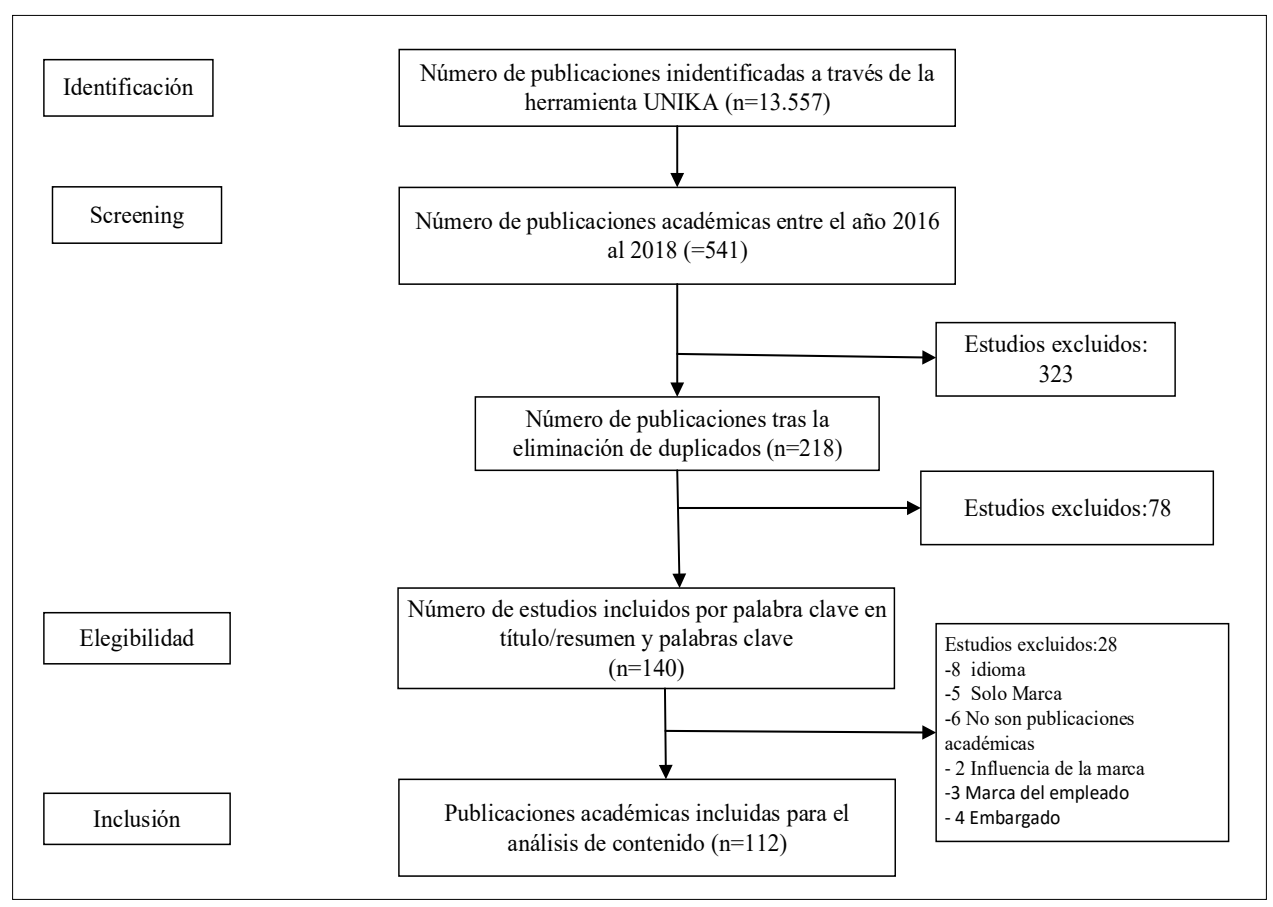

Fuente: elaboración propia (Diagrama de flujo PRISMA)

Para asegurar que la publicación pertenecía al universo de estudio deseado, una vez identificadas las publicaciones, el siguiente paso consistió en limitarlas a aquellas que incluían los términos en el título, el resumen y/o palabras clave. De este modo, la muestra se redujo a 140 publicaciones (ver figura 1) que se esperaba tratasen la marca corporativa de manera destacada.

Una revisión individualizada de los trabajos permitió detectar 10 casos en los que, a pesar de usar el término, realmente no abordaban la marca corporativa. Se excluyeron otros 6 trabajos por no tratarse de publicaciones académicas y 12 más por no permitir su lectura por estar embargado y/o porque su texto completo no estaba en inglés o castellano (solo lo es- 
taba el resumen). Tras aplicar estos criterios de inclusión y exclusión, se identificaron finalmente 112 unidades de análisis (ver figura 1).

Una vez seleccionada la muestra, los nombres de las revistas donde aparecían las publicaciones se anotaron en hojas de cálculo, obteniendo un total de 66 revistas distintas. A partir de este listado, se identificaron la disciplina o disciplinas en las que se ubican las revistas.

En la identificación se utilizó la clasificación que otorga el Scimago Journal \& Country Rank. Se utilizó el SJR por ser un portal que incluye revistas e indicadores científicos a partir de la información contenida en la base de datos Scopus (Elsevier BV). Esta permite clasificar las revistas por área temática (27 áreas temáticas principales), categoría temática (313 categorías temáticas específicas) o por país (SJR, 2020). Del total de 66 revistas clasificadas, 18 no aparecían en la base de datos del SJR. En esos casos, se les asignó el área o áreas temáticas que indicaban las páginas web de las respectivas revistas.

La segunda parte del trabajo consistió en la revisión completa de las 112 publicaciones registradas para identificar autor, presencia de otros términos relacionados con la marca corporativa como identidad corporativa e imagen corporativa y su vinculación, así como la identificación o no del responsable o área donde se ubica su gestión.

A partir de esta lectura, se determinaron además 8 ámbitos en los que pueden clasificarse los trabajos encontrados, atendiendo al contenido central que se trata en ellos:

1. Conceptualización de marca corporativa

2. Construcción de marca corporativa

3. Gestión de marca corporativa

4. Identidad de marca corporativa

5. Imagen de marca corporativa

6. Marca corporativa y relación con stakeholders

7. Reputación de marca corporativa

8. Valor de marca corporativa

Esta clasificación se utilizó para ubicar a los autores encontrados, lo que ha servido para detectar los más importantes en cada una de ellas (ver tabla 4).

\section{Resultados}

Tal y como se ha señalado, los 112 artículos identificados fueron publicados en 66 revistas. El 54\% (61) fue publicado en 15 de esas 66 revistas (ver anexo 2), destacando el Journal of Product and Brand Management con 15 artículos relacionados con la marca corporativa, incluida una edición especial en el volumen 27 de 2018 sobre branding corporativo (ver tabla 1). 
Tabla 1. Revistas con al menos dos artículos

\begin{tabular}{|l|c|}
\hline \multicolumn{1}{|c|}{ Revistas 2016-2018 } & $\begin{array}{c}\mathbf{N}^{\circ} \text { de artículos } \\
\text { publicados }\end{array}$ \\
\hline Journal of Product \& Brand Management & 15 \\
\hline Journal of Brand management & 10 \\
\hline European Journal of Marketing & 5 \\
\hline International Studies of Management \& Organization & 5 \\
\hline Journal of Business Research & 5 \\
\hline Journal of Strategic Marketing & 3 \\
\hline Asia Pacific Journal of Marketing and Logistics & 2 \\
\hline Business ethics quarterly & 2 \\
\hline Corporate Reputation Review & 2 \\
\hline International Journal of Bank Marketing & 2 \\
\hline International Marketing Review & 2 \\
\hline Journal of Business Ethics & 2 \\
\hline Marketing Intelligence \& Planning & 2 \\
\hline Profesional de la información & 2 \\
\hline Revista Latina de Comunicación & 2 \\
\hline Total & $\mathbf{5 4}$ 5\% de los 112 articulos \\
\hline
\end{tabular}

Fuente: elaboración propia

El Journal of Brand Management es la segunda revista con más publicaciones realizadas entre 2016 y 2018, con 10 artículos. Esta revista, junto al European Journal of Marketing ha publicado el mayor número de trabajos relacionados con la marca corporativa desde el año 2000, tal y como demuestra el estudio de Mingione (2015).

Las 12 revistas restantes publicaron de dos a cinco artículos (ver tabla 1). Al igual que en el estudio de Mingione (2015), la presencia de revistas procedentes del área de la comunicación es escasa.

Las dos revistas que publicaron más artículos tienen como área temática a Business, Management and Accountingy, como categoría, el Marketing (ver anexo 2). Sin embargo, el análisis de los 112 trabajos y las 66 revistas permite identificar más de 20 áreas temáticas, muchas compartidas, al igual que las dos citadas. 


\subsection{Disciplinas donde se ubican los trabajos y el rol de la comunicación}

Para determinar qué importancia tiene cada área en el conjunto de la muestra, se analizó el listado de todas las áreas temáticas (ver anexo 3) mediante el recuento de palabras. Utilizando la aplicación WordCounter se identificó que el área temática de Business, Management and Accounting es la más destacada entre un total de 21 disciplinas posibles y no excluyentes. Si tenemos en cuenta las 66 revistas, 41 se vinculan a este conjunto temático (ver gráfico 1). Sobre el total de 112 trabajos, 83 (el 74\%) se clasificarían en este grupo.

Dentro del área temática de Business, Management and Accounting, se encuentra la categoría del Marketing, con 18 revistas y 55 publicaciones. Las dos revistas que publicaron más artículos se clasificaban en la misma categoría. Si sumamos la temática y la categoría de Marketing, encontramos un total de 59 publicaciones (53\% de las 112 clasificadas), por lo que la relevancia del área de Marketing para el estudio y análisis de la marca corporativa parece indiscutible.

La segunda área temática con mayor presencia en el listado de disciplinas (anexo 3) es Ciencias Sociales, con 13 revistas (ver gráfico 1). Dentro de esta área temática se clasifica la categoría de la Comunicación, con una revista y dos publicaciones. De las 66 revistas, solo dos se vinculan a Comunicación como área temática, con tres artículos de los 112 clasificados, lo que da un total de cinco artículos procedentes del ámbito de la comunicación.

Gráfico 1. Las cinco primeras áreas temáticas de las revistas*

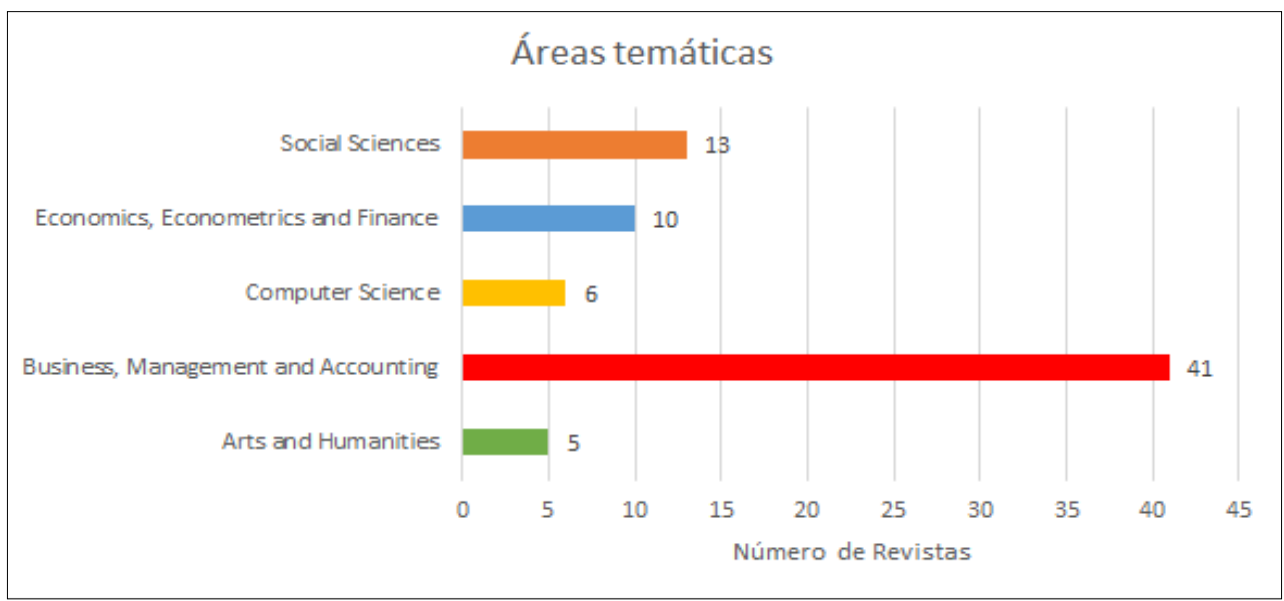

*El total de revistas es de 66, aunque pueden vincularse a más de un área temática.

Fuente: Recuento elaborado con la aplicación WordCounter, según las temáticas identificadas por el Scimago Journal \& Country Rank o las páginas web de las revistas. 
En tercer lugar, encontramos el área de Economía, Econometría y Finanzas: 13 revistas con 15 artículos comparten esta temática (ver anexo 3). Una cuarta área temática con trabajos sobre marca corporativa es la de Ciencias de la Computación. Y en quinto lugar destacamos el área temática de Artes y Humanidades, compartida por cinco revistas que también se incluyen en el área de Business, Management and Accounting.

Además de estas cinco áreas temáticas, que engloban al menos 40 de las 66 revistas (ver gráfico 1), hay otras 16 áreas temáticas compartidas por 22 revistas (ver anexo 2). Nos encontramos así con una alta variedad temática, destacando las revistas de las disciplinas de la gestión y el marketing, pero no de la comunicación.

\subsection{Autores destacados en el estudio de la marca corporativa}

Si atendemos a los autores citados en los trabajos sobre marca corporativa en vez de a las áreas temáticas, es posible detectar algunos referentes destacados. El investigador más citado en las publicaciones clasificadas (2016-2018) para conceptualizar la marca corporativa, su gestión o los valores, entre otros ámbitos, es John M.T. Balmer (ver tabla 2).

Tabla 2. Los cinco autores más citados en las 112 publicaciones

\begin{tabular}{|l|c|c|}
\hline $\begin{array}{c}\text { Autores más citados } \\
\text { en las publicaciones }\end{array}$ & $\begin{array}{c}\mathbf{N}^{\circ} \text { de } \\
\text { publicaciones } \\
\text { citados }\end{array}$ & $\begin{array}{c}\% \text { de citas en las } \\
112 \\
\text { publicaciones }\end{array}$ \\
\hline Balmer & 55 & $49,1 \%$ \\
\hline De Chernatony & 25 & $22,3 \%$ \\
\hline Hatch and Schultz & 24 & $21,4 \%$ \\
\hline Keller & 22 & $19,7 \%$ \\
\hline Aaker & 21 & $18,8 \%$ \\
\hline
\end{tabular}

Fuente: elaboración propia

De las 112 publicaciones analizadas, 55 citan a John M.T. Balmer. Además, él mismo es autor de siete artículos relacionados con la marca corporativa en el periodo de estudio (Balmer, Abratt, \& Kleyn, 2016; Balmer \& Chen, 2016; Balmer, 2017; Balmer \& Wang, 2016b; Santos, Burghausen, \& Balmer, 2016). 
Tabla 3. Autores y ámbitos desarrollados en las publicaciones

\begin{tabular}{|c|c|}
\hline Ámbitos temáticos & Autores \\
\hline $\begin{array}{l}\text { Conceptualización } \\
\text { marca corporativa }\end{array}$ & $\begin{array}{l}\text { Aaker (2004); Aaker y Joachimsthaler (1999, 2000, 2012); Abratt y Kleyn (2012); Argenti, Druckenmiller y Novelli (2004); } \\
\text { Balmer (1995, 1998, 2001, 2003, 2008, 2009, 2010, 2011,2012, 2013); Balmer y Gray (2003); Balmer y Greyser (2002, 2003, } \\
\text { 2006); Balmer y Thomson (2009); Biraghi y Gambetti (2015); Burmann et al. (2009); De Chernatony (2002); De Chernatony } \\
\text { y Dall'Olmo Riley (1998); Christensen, Morsing y Cheney (2008); Cornelissen et al.; (2007); Cornelissen, Christensen y } \\
\text { Kinuthia(2012); Fetscherin y Usunier (2012); Foster et al. (2010); Grifin (2002); Halliday (1998); Harris y de Chernatony } \\
\text { (2001); Hatch y Schultz (2001, 2003, 2009); Ind (1997, 1998); Kapferer (2002); Kay (2006); Keller (1998, 2003, 2008); Keller } \\
\text { y Lehmann (2006); Keller y Richey (2006); King (1991); Knox y Bickerton (2003); Laine (2011); Leitch \& Richardson (2003); } \\
\text { Mann y Ghuman (2014); Melewar y Jenkins (2002); Merz, He y Vargo, (2009);Miyazaki et al. (2005); Morsing (2006); Olins } \\
\text { (2000); Pillai (2012); Pratihari y Uzma (2018); Rao, Agarwal \& Dahlhoff (2004); Rao y Ruekert (1994); So (2013); Schmidt \& } \\
\text { Redler (2018); Schultz, Antorini y Csaba (2005); Schultz y Chernatony (2002); Schultz (2005); Urde (2003, 2007, 2013); Van } \\
\text { Riel (2001);Wiedmann (2015). }\end{array}$ \\
\hline $\begin{array}{l}\text { Construcción de } \\
\text { marca corporativa }\end{array}$ & $\begin{array}{l}\text { Aaker (2004), Andreassen y Lindestad (1998); Anisimova (2007); Balmer (2009, 2012); Ballantyne y Aitken (2007); Biraghi y } \\
\text { Gambetti (2015); Brown y Dacin (1997); Brown, Dacin, Pratt \& Whetten (2006); Cornelissen, Christensen, y Kinuthia (2012); } \\
\text { Daffey y Abratt (2002); Dacin y Brown (2002); Edvardsson et al. (2011); Einwiller y Will (2002); Franklin (2008); Fetscherin } \\
\text { y Usunier (2012);Fombrun y Shanley (1990); Hatch and Schultz (2001, 2003, 2010); Hulberg (2006); Keller (2001); Keller y } \\
\text { Aaker (1998); Knox y Bickerton (2003); Pratihari y Uzma (2018); Rubin (2012); Schultz et al. (2005); So (2013); Srinivasan et } \\
\text { al. (2010); Stuart (2011); Urtde, et al. (2011); Vallaster et al. (2012); Worcester (2009). }\end{array}$ \\
\hline $\begin{array}{l}\text { Gestión de marca } \\
\text { corporativa }\end{array}$ & $\begin{array}{l}\text { Aaker (2004); Abratt y Kleyn (2012); Balmer (1995, 2001,2008,2011, 2012,2013); Balmer et al., (2017); Balmer y Greyser (2003); } \\
\text { Balmer y Soenen, (1999); Balmer and Wilkinson (1991); Christensen y Cornelissen (2011); Einwiller y Will (2002); Fetscherin } \\
\text { y Usunier (2012); Hatch y Schultz (2009, 2010); Harris y de Chernatony (2001); He y Balmer (2006); Helm y Jones (2010); Ind } \\
\text { et al. (2013); Jaju et al. (2006); Juntunen (2012); Knox (2004); Knox y Bickerton (2003); Leitch y Richardson (2003); Merrilees } \\
\text { (2016); Merrilees y Miller (2008); Melewar } \text { et al. (2012); Muzellec y Lambkin (2008); Punjaisri y Wilson (2011); Shahri (2011); } \\
\text { Strebinger (2014); Uggla (2006). }\end{array}$ \\
\hline $\begin{array}{l}\text { Identidad de } \\
\text { marca corporativa }\end{array}$ & $\begin{array}{l}\text { Aaker (2004); Aaker y Joachimsthaler (2012);Abratt (1989); Balmer (2001, 2008, 2010, 2011, 2012); Balmer y Burghausen } \\
\text { (2015); Balmer y Gray (2003);Balmer y Greyser (2002); Baumgarth (2010); Blombäck \& Ramírez-Pasillas (2012); Cornelissen } \\
\text { et al. (2012); Currás, (2010); Christensen \& Cornelissen (2010); Edvardsson, Tronvoll, \& Gruber (2011); Gryd-Jones et al. } \\
\text { (2013); Harris \& de Chernatony (2001); Hatch \& Schultz (2001); Heding, Knudtzen y Bjerre (2008); Hur et al. (2014); Kapferer } \\
\text { (2002; 2012); Keller (2008); Loe, Ferrell y Mansfield (2000); Melewar y Jenkins (2002); Melewar (2003); Merz et al. (2009); } \\
\text { Mulki y Jaramillo (2011); Petkova et al. (2008); Simões et al. (2005); Silveira et al. (2013); Schultz et al. (2005); Urde (1999, } \\
\text { 2013); Urde (2013); Urde, Baumgarth, \& Merrilees, (2013); Urde y Greyser (2015, 2016); Witt \& Rode (2005). }\end{array}$ \\
\hline $\begin{array}{l}\text { Imagen de marca } \\
\text { corporativa }\end{array}$ & $\begin{array}{l}\text { Aaker (1996); Balmer (1995); Blombäck y Axelsson (2007); Chi-Shiun et al. (2010); Cretu y Brodie (2007); De Chernatony } \\
\text { (1999); Dennis et al. (2007); Hatch \& Schultz, (2003) Ind (1997); Leitch \& Richardson (2003); Muzellec and Lambkin, (2009); } \\
\text { Torelli et al. (2012); Wiedmann et al. (2011). }\end{array}$ \\
\hline $\begin{array}{l}\text { Marca corporativa } \\
\text { y relación con } \\
\text { stakeholders }\end{array}$ & $\begin{array}{l}\text { Abratt y Kleyn (2012);Anisimova (2007);Argenti y Druckenmiller (2004);Balmer (1995, 2001;2005, 2010; 2013); Balmer y Gray } \\
\text { (2003);Balmer y Greyser, (2002, 2006); Balmer and Wilkinson (1991); Balmer et al. (2017); Bergami y Bagozzi (2000);Biraghi } \\
\text { \& Gambetti (2015); Bhattacharya et al.(2009);Brown and Dacin (1997); de Chernatony (2001,2002); de Chernatony y } \\
\text { Harris(2000); Fatma y Rahman (2015); Guglery Shi (2009); Gyrd-Jones \& Kornum (2013);Harris y deChernatony (2001); Hatch } \\
\text { y Schultz (2003);Ind (2001); Janssen et al. (2012); Kaufmann et al. (2012); Knox and Bickerton (2003); Leitch y Richardson } \\
\text { (2003); Melewar (2003); Merz, He y Vargo,(2009).; Oberseder } \text { et al. (2013); Papasolomou y Demetriou (2005);Podnar (2015); } \\
\text { Podnar,et al.(2011); Polonsky y Jevons (2006);Roper y Davis (2007); Rubin (2012); Schultz, (2005); (Schwaiger \& Sarstedt } \\
\text { (2011); Suvatjis y de Chernatony (2005); Veselinova, et al. (2015). }\end{array}$ \\
\hline $\begin{array}{l}\text { Reputación de } \\
\text { marca corporativa }\end{array}$ & $\begin{array}{l}\text { Abratt y Kleyn (2011); Anisimova (2010); Balmer (2001, 2011, 2012); Balmer y Gray (2003); Boulding (1956); Boyd et al. } \\
\text { (2010); Chabowski } \text { et al. (2011); De Chernatony y Harris (2000); Deephouse (2000); Erdem, Erdum \& Swait (1998); Fombrun } \\
\text { (1996); Gatewood, Gowan y Lautenschlager (1993);Glynn (2012); Gomes et al. (2016); Gruning (1993); Jung (2011); Hatch y } \\
\text { Schultz (2008); Hur, et al. (2014); Low, Blois (2002); Sweetin et al. (2013); Roberts y Dowling (2002); Roper y Fill (2012); Urde } \\
\text { y Koch (2014); Van Riel y Balmer (1997); Voss \& Mohan (2016); Weun et al. (2004); Zuckerman \& Kim (2003). }\end{array}$ \\
\hline $\begin{array}{l}\text { Valor de marca } \\
\text { corporativa }\end{array}$ & $\begin{array}{l}\text { Aaker (2004); Abratt y Kleyn (2011, 2012); Argenti y Druckenmiller (2004); Balmer (2001, 2008, 2010, 2011); Balmer y Greyser } \\
\text { (2003); Balmer y Gray (2003); Chang y Ko (2014); Chang, et al. (2009); Hamzah, et al. (2014); Hatch y Schultz (2003); Harris } \\
\text { y De Chernatony (2001); Heinberg et al. (2018); Hur et al. (2014); Hsu (2012); Lynch, de Chernatony (2007); Lin (2013); } \\
\text { Malcolm y Driesener (2016); Rao et al. (2004); Shahri (2011); Stanaland, Lwin, \& Murphy (2011); Trauer y Ryan (2005); Urde } \\
\text { (2003); Urde y Koch (2014); Uggla (2006); Uriely, Yonay y Simchai (2002); Van Riel, De Mortanges, Streukens (2005); Wang \& } \\
\text { Sengupta (2016). }\end{array}$ \\
\hline
\end{tabular}

Fuente: elaboración propia 
Cabe apuntar que, de las 112 publicaciones identificadas, 13 de ellas no citaron a ningún autor vinculado al concepto de marca corporativa. Esto hace que el papel de John M.T. Balmer resalte aún más: de las 99 publicaciones con referencias, le citan más de la mitad (un 55,5\% de los trabajos). Si atendemos a los ámbitos temáticos de los trabajos de Balmer (ver tabla 3), podemos observar que es uno de los pocos autores que aporta publicaciones en todos ellos. Además de ocuparse de la conceptualización de la marca corporativa, ha aportado sobre su construcción y gestión, y estudiado la identidad, la imagen, la relación de la marca con los stakeholders, la reputación o el valor de marca.

El segundo autor destacado es De Chernatony citado en 25 artículos, además de contribuir al campo con diferentes investigadores (Dall'Olmo Riley \& de Chernatony, 2000; Harris \& de Chernatony, 2001; Schultz \& de Chernatony, 2002). También es un autor que ha sido citado en todos los ámbitos destacados, salvo el correspondiente a la construcción de la marca corporativa (ver tabla 3 ).

El tercer lugar lo ocupan las autoras Jo Hatch \& Schultz, que fueron citadas en 24 artículos (ver tabla 2). Estas académicas han aportado al constructo teórico de la marca corporativa en todas las áreas, salvo en la conceptualización de la marca corporativa, ya que no se identificaron citas relacionadas en los trabajos analizados (ver tabla 3).

Respecto a las áreas donde se ubican estos autores, la comunicación no aparece como tal en sus currículos. John M.T. Balmer indica que sus campos de investigación son el marketing corporativo y la gestión de marca e identidad corporativas. Leslie de Chernatony, profesor de “Brand Marketing”, destaca la gestión de marca como su campo de interés. Mary Jo Hatch enmarca su investigación en la gestión, el marketing y la teoría de las organizaciones. Finalmente, Majken Schultz señala como sus tres principales áreas de investigación la identidad de la organización, la marca corporativa y la cultura organizacional.

En resumen, los cuatro autores más citados en los trabajos sobre marca corporativa muestran un mayor interés por investigar en el área de la gestión o el marketing que en el área de la comunicación. En todo caso, debemos tener en cuenta que todos ellos están vinculados a escuelas de negocio, lo que explicaría su foco en la gestión.

\subsection{Conceptos vinculados}

Un análisis de los contenidos de las publicaciones nos permite situar el origen del concepto de la marca corporativa en la diferenciación entre la marca de producto y la marca corporativa, términos que difieren en su alcance y gestión disciplinaria (Balmer, 1995, 2001a; Balmer \& Gray, 2003; King, 1991; Schultz et al., 2005). Algunos trabajos establecen que la marca de producto representa un servicio de un solo productor, mientras que la marca corporativa hace referencia a toda la organización, con una perspectiva más estratégica (Balmer, 2001b; Balmer \& Gray, 2003).

Sin embargo, el desarrollo de la marca corporativa vino de la mano de la imagen corporativa y la identidad corporativa (Schmidt \& Redler, 2018: 189). Los trabajos del ámbito definido como "identidad de marca corporativa" indican que la marca puede considerarse un tipo de identidad distinta que tiene vida propia (Balmer, 1995; 2001b); que se describe como la expresión de la identidad de una organización (Abratt \& Kleyn, 2012), a través de la cual se define la empresa y es la encargada de respaldar la oferta que el cliente comprará (Aaker, 2004). 
La articulación de la identidad es un requisito para el éxito de la construcción de la marca corporativa (Stuart, 2011). A través de la articulación de la identidad se puede obtener la preferencia de los clientes hacia la organización y así, lograr una ventaja competitiva (Anisimova, 2016; Daffey \& Abratt, 2002; Keller \& Aaker, 1998).

Autores como Törmälä \& Gyrd-Jones (2017) señalan que se puede acceder a la identidad de marca corporativa preguntando: “¿Quiénes somos como empresa? (Balmer, 2001a; Melewar \& Jenkins, 2002), y: “¿Cómo deseamos que nos perciban los interesados?" (Aaker \& Joachimsthaler, 2000; Alwi, Ali, \& Nguyen, 2017). La importancia de la identidad de marca corporativa se refleja en el hecho de que las empresas necesitan dirigirse a los consumidores y a otras partes interesadas mediante la construcción de dicha identidad, la imagen y la reputación corporativa (Alwi et al., 2017).

Otro de los conceptos que los trabajos sobre marca corporativa estudian es, el de "imagen de marca corporativa” y no así el de "imagen corporativa". Desde su perspectiva, se destaca que la marca corporativa representa más que la manifestación externa de una organización, es decir, más que su nombre, logotipo o código visual. La marca corporativa personifica, simbólicamente hablando, la representación de los valores subyacentes de la organización (Balmer \& Gray, 2003b; Ind, 1997). Como vimos anteriormente, Knox \& Bickerton (2003) también la definen como una expresión visual, verbal y de comportamiento.

La definición de imagen de marca corporativa es importante para desarrollar el propio concepto y desarrollo de la marca corporativa. La imagen corporativa de la marca constituye un conjunto de percepciones externas o asociaciones mentales sobre la totalidad de la compañía (Balmer, 2001b; Balmer; Gray, 2003; Brown; Dacin; Pratt; Whetten, 2006), que se construyen con el tiempo en función de las experiencias de los clientes con la marca de la empresa (Wiedmann, Hennigs, Schmidt, \& Wuestefeld, 2011).

Pero además de los ámbitos de la identidad o la imagen, algunos trabajos se engloban en el estudio de la relación de los stakeholders con la marca corporativa. Todos ellos se encuentran involucrados en la creación y, en ocasiones, en la administración de las marcas corporativas (Balmer \&Wang, 2016a). Por ello, las relaciones con los stakeholders pueden contribuir a dar forma a la marca corporativa de la empresa (Schwaiger \& Sarstedt, 2011). A pesar de hablarse de stakeholders, llama la atención que solo uno de los 112 trabajos menciona el área de las relaciones públicas (Erdal, 2017).

Finalmente, la reputación y el valor de marca corporativa son otras dos cuestiones abordadas en las publicaciones analizadas. Dentro de este ámbito, nuevamente se identificó tan solo un trabajo que destaca el papel de las relaciones públicas (Heller, 2016). Esta publicación remarca su contribución a la creación de estrategias que destacan las actividades de las organizaciones para mejorar la reputación corporativa y la reputación de la marca corporativa.

En resumen, encontramos ligados a marca corporativa los conceptos de marca en general, pero también otros como identidad de marca corporativa, imagen de marca corporativa, stakeholders y reputación, asociados a comunicación institucional o relaciones públicas. Sin embargo, solo dos trabajos destacan el papel de estas a la hora de promover relaciones entre los stakeholders con las empresas o mejorar la reputación. 


\subsection{La gestión de la marca corporativa}

Al estudiar las referencias a las áreas de gestión vinculadas a la marca corporativa en el conjunto de los trabajos estudiados, se observa que no todas las publicaciones analizadas mencionan a quién corresponde tal gestión. Sí lo hacen 69 de las 112 publicaciones (ver gráfico 2).

Gráfico 2. ¿Desde qué áreas se debe gestionar la marca corporativa?

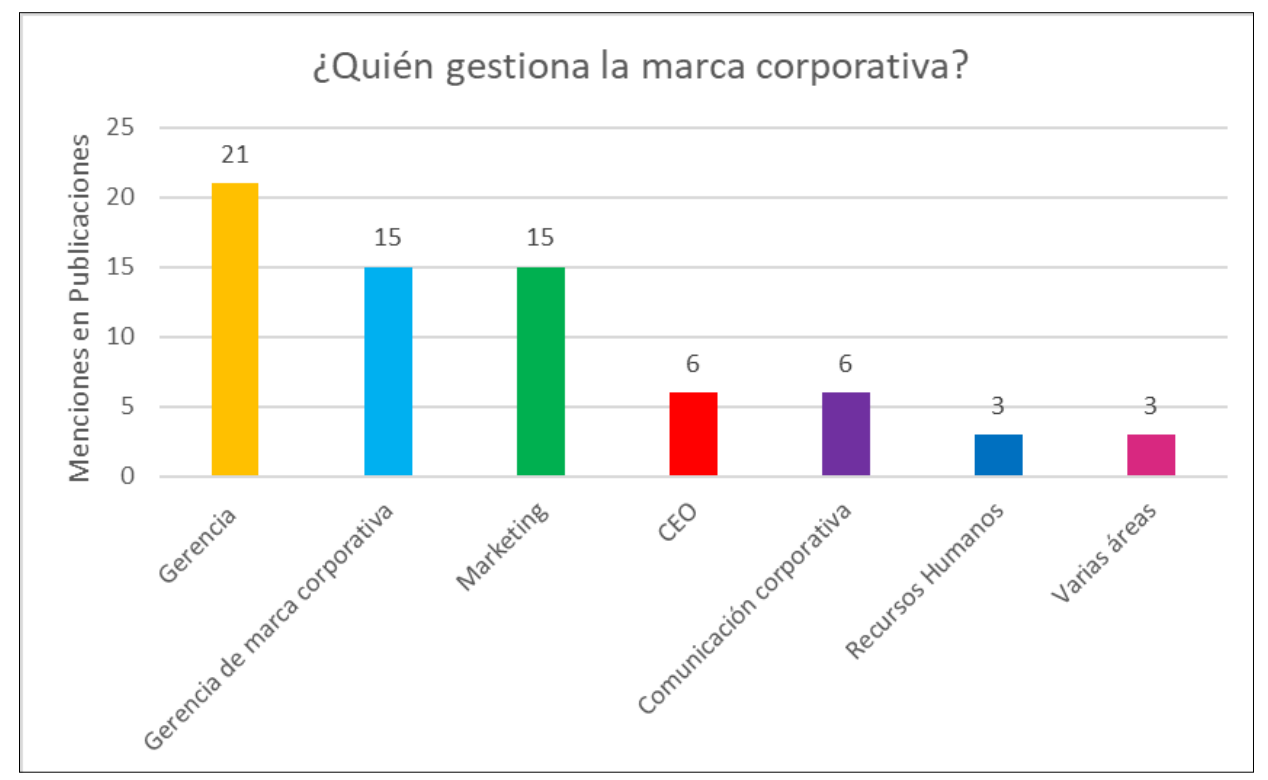

Fuente: elaboración propia

El área más destacada es la gerencia (senior management), la cual ha sido identificada como la responsable directa de la gestión de la marca corporativa en 21 artículos. La referencia explícita al CEO de la empresa se realiza en seis publicaciones (ver gráfico 2).

En segundo lugar, la gestión de la marca corporativa se atribuye a la gerencia de marca corporativa, mencionada en 15 de las 112 publicaciones. La tercera área de gestión mencionada es el marketing, que aparece en 15 artículos (ver gráfico 2).

Si analizamos los trabajos que enmarcan la gestión de la marca corporativa en comunicación, observamos que esta se considera el área encargada de gestionar únicamente los canales y herramientas de comunicación que la organización utiliza para comunicarse con los stakeholders (Balmer, 2001a). No hacen referencia a su valor como activo estratégico.

Otra área mencionada al hablar de la gestión de la marca corporativa es recursos humanos, con tres artículos (Buil, Catalán, \& Martínez, 2016; Markovic Markovic, Iglesias Bedós, Singh, \& Sierra Olivera, 2018). Sin embargo, no se cita como área 
para su gestión, sino como elemento que debe ser considerado por este departamento, alineando la estrategia de marca corporativa con las políticas y prácticas de recursos humanos.

En resumen, no existe consenso en cuanto al área o áreas que deben encargarse de la gestión de la marca corporativa. Sin embargo, al ser un activo estratégico que deriva de la identidad corporativa, destacan las referencias a la gerencia y el apoyo que requiere de todos los miembros de la organización.

\section{Discusión y conclusiones}

En este trabajo se constata que el desarrollo y estudio de la marca corporativa no es terreno de una sola disciplina, como ya habían indicado otros estudios (Balmer \& Gray, 2003; Fetscherin \& Usunier, 2012; King, 1991). En el estudio de 112 trabajos publicados de 2016 a 2018, se identifican hasta 20 disciplinas donde se clasifican las 66 revistas que incluyen estas obras.

Las dos disciplinas destacadas son gestión y marketing. Estas han reconocido la importancia de la marca corporativa y su papel como activo para conseguir la diferenciación frente a la homogeneización de productos y la globalización, contribuyendo de manera destacada al desarrollo teórico del área. También se ve como una parte cada vez más importante, poderosa y visible de la economía (Balmer, 2001b; Urde, Greyser, \& Balmer, 2007) de las organizaciones.

La marca corporativa se ha estudiado incluso desde las ciencias de la computación, con estudios de posicionamiento o basados en la percepción de los clientes. Desde el área temática de las Artes y Humanidades, se discute acerca de cómo la imagen ética puede construir equidad en las marcas de servicios corporativos (Sierra, Iglesias, Markovic, \& Singh, 2017), o cómo el patrimonio negativo puede afectar al desarrollo de las marcas de patrimonio corporativo (Sørensen, Korsager, \& Heller, 2018).

Diversos autores han subrayado la importancia de la experiencia del consumidor y las relaciones con los diferentes stakeholders en la creación de valor de marca corporativa y la mejora de su reputación (Balmer,1995; Jo Hatch \& Schultz, 2003b; Schmidt \& Redler, 2018). Sin embargo, en el presente estudio, tan solo dos trabajos destacan el papel de las relaciones públicas a la hora de promover relaciones de los stakeholders con las empresas y la importancia que ello conlleva para la gestión de la marca corporativa. En este punto, resulta llamativa la ausencia de mayores referencias en este campo, puesto que las teorías de relaciones públicas, como la teoría situacional de los públicos, podrían constituir una aproximación más que complementaria al estudio de las relaciones con los diversos stakeholders en la gestión de la marca corporativa.

La comunicación tampoco es el campo donde se ubican los autores que más han contribuido al desarrollo de la marca corporativa en el periodo 2016-2018. Los cuatro autores más citados en los 112 trabajos estudiados son John M.T. Balmer, Leslie de Chernatony, Mary Jo Hatch y Majken Schultz. Todos ellos trabajan en escuelas de negocio y sus investigaciones se centran en la gestión y el marketing. Vinculado a la comunicación destacaría solo van Riel, citado en 11 artículos $(9,8 \%)$ cuando el resto de los autores aparecen en más de 20.

Respecto a su gestión, si analizamos quién es el responsable, la comunicación tampoco parece que esté jugando un papel clave. Aunque en algún momento se consideró la marca corporativa como una cuestión de los departamentos de comu- 
nicación corporativa (Balmer, 2008a; Biraghi; Gambetti, 2015), nuestro estudio destaca el papel de la gerencia y CEOs de la empresa a la hora de diseñar e implementar una estrategia de marca corporativa. Autores como Balmer \&Wang (2016a) así lo confirman, enfatizando la importancia y las obligaciones de los gerentes sénior y del CEO en la gestión de las marcas corporativas.

Otra área posible es la de marketing corporativo (Balmer, 2001a). Esta se encargaría de mantener relaciones continuas con los clientes y el resto de stakeholders a través de la marca corporativa (Balmer \& Greyser, 2006; Balmer, 2017). Se trataría de transferir la asociación de la marca corporativa a las submarcas (Brexendorf \& Keller, 2017). Por tanto, la congruencia entre la identidad de marca deseada y percibida es parte del trabajo del gerente de marca corporativa (Abratt \& Kleyn, 2012). En este sentido, Sevel et al. (2018) apuntan que la gerencia de marca corporativa requerirá el apoyo y consenso de los demás miembros y áreas de la organización, lo que nos lleva a la necesidad de ocupar un lugar destacado en lo alto del organigrama de la organización.

La gestión de la marca corporativa requiere una comprensión que involucre a todos los stakeholders de la organización, un manejo cuidadoso de los elementos que componen la identidad (Dall'Olmo Riley \& de Chernatony, 2000), la cultura organizacional y que represente los valores de la organización. Por ello, parece difícil que los departamentos de comunicación corporativa puedan crear y desarrollar por sí solos una marca sólida (Kay, 2006) que logre responder a todas las necesidades de los diferentes stakeholders.

En consonancia con Cornelissen (2008), consideramos que la gestión de la marca corporativa requiere de las aportaciones de distintas áreas de las empresas, entre ellas, el marketing. Se trata de lograr una visión amplia e integradora, que cuente con el apoyo y compromiso desde las esferas más altas de la organización, el CEO (Balmer; Gray, 2003; Uggla, 2006; Balmer, 2008b; Shahri, 2011) y de un enfoque que integre a todos los stakeholders (Balmer \& Wang, 2016a; Dunnion \& Knox, 2004; Vallaster, Lindgreen, \& Maon, 2012).

En conclusión, la gestión de la marca corporativa, como activo estratégico, debe contar con una intención estratégica a largo plazo, enfocada en la construcción de una marca corporativa sólida y reconocible, que respalde toda la cartera de productos de la empresa. A través de ella se pueden crear asociaciones favorables hacia la compañía y responder así a un contexto cambiante, y a todos los públicos implicados. Por consiguiente, la gestión de la marca, corporativa o no, no debería estudiarse de forma aislada, sino que habría que considerarla más bien como un activo estratégico.

Se trata de gestionar una marca corporativa que comunica, diferencia y posiciona a la organización. Esto implica que, en el diseño de la estrategia de marca corporativa, se requiere la implicación de todas las áreas de comunicación corporativa, interna y externa, junto a las áreas de marketing, recursos humanos y negocios de manera sinérgica, holística y coordinada. Desde el punto de vista de la academia, esto supone que tienen sentido las aportaciones sobre la marca de todos estos ámbitos, recomendándose una visión multidisciplinar. 


\section{Referencias bibliográficas}

Aaker, D. A. (1991). Managing Brand Equity: Capitalizing on the Value of a Brand Name. New York: Free Press. https://doi. org $/ 10.2307 / 1252048$

Aaker, D. A. (2004). Leveraging the Corporate Brand. California management review, 46(3), 1-14.

Aaker, D. A., \& Joachimsthaler, E. (2000). The Brand Relationship Spectrum: The key to the brand architecture challenge. California Management Review, 42(4), 8-23. Retrieved from https://eds.a.ebscohost.com/eds/pdfviewer/ pdfviewer?vid=1\&sid=8b0f45e6-88f4-47e5-8c9a-28e138794a27\%40sdc-v-sessmgr04

Abratt, R., \& Kleyn, N. (2012). Corporate identity, corporate branding and corporate reputations: Reconciliation and integration. European Journal of Marketing, 46(7/8), 1048-1063. https://doi.org/10.1108/03090561211230197

Alwi, S. F. S., Ali, S. M., \& Nguyen, B. (2017). The Importance of Ethics in Branding: Mediating Effects of Ethical Branding on Company Reputation and Brand Loyalty. Business Ethics Quarterly, 27(3), 393-422. https://doi.org/10.1017/beq.2017.20

Anisimova, T. (2016). The effects of corporate brand symbolism on consumer satisfaction and loyalty: Evidence from Australia. Asia Pacific Journal of Marketing and Logistics, 28(3), 481-498. https://doi.org/10.1108/APJML-05-2015-0086

Argenti, P. A., \& Druckenmiller, B. (2004). Reputation and the Corporate Brand. Corporate Reputation Review, 6(4), 368374. https://doi.org/10.1057/palgrave.crr.1540005

Balmer, J. M. T., \& Soenen, G. B. (1997). Operationalising the Concept of Corporate Identity: Articulating the Corporate Identity Mix and the Corporate Identity Management Mix. (D. of M. University of Strathclyde, Ed.). Glasgow. Retrieved from https://eds.a.ebscohost.com/eds/detail/detail?vid=3\&sid=8599e69a-bd28-4386-9blf-180d7cc28fbf\%40sdc-v-sessmgr03\&bdata=JkF1dGhUeXBlPWlwLHVybCZsYW5nPWVzJnNpdGU9ZWRzLWxpdmUmc2NvcGU9c210ZQ\%3D\%3D\#AN=edseul.3000076240645\&db=edseul

Balmer, J. M. T. (1995). Corporate Branding and Connoisseurship. Journal of General Management, 21(1), 24-46. https:// doi.org/10.1177/030630709502100102

Balmer, J. M. T. (1998). Corporate Identity and the Advent of Corporate Marketing. Journal of Marketing Management, 14(8), 963-996. https://doi.org/10.1362/026725798784867536

Balmer, J. M. T. (2001a). Corporate identity, corporate branding and corporate marketing Seeing through the fog. European Journal of Marketing, 35(3/4), 248-291. https://doi.org/10.1108/03090560110694763

Balmer, J. M. T. (2001b). The Three Virtues and Seven Deadly Sins of Corporate Brand Management. Journal of General Management. https://doi.org/10.1177/030630700102700101

Balmer, J. M. T. (2008a). An epiphany of three: corporate identity, corporate brand management, and corporate marketing. In Routledge (Ed.), Facets of corporate Identity, Communication and reputation (pp. 35-54). Abingdon, UK: Taylor \& Francis Group. 
Balmer, J. M. T. (2008b). Identity based views of the corporation: Insights from corporate identity, organisational identity, social identity, visual identity, corporate brand identity and corporate image. European Journal of Marketing, 42(9/10), 879-906. https://doi.org/10.1108/03090560810891055

Balmer, J. M. T. (2010). Explicating corporate brands and their management: Reflections and directions from 1995. Journal of Brand Management, 18(3), 180-196. https://doi.org/10.1057/bm.2010.46

Balmer, J. M. T. (2011). Corporate marketing myopia and the inexorable rise of a corporate marketing logic: Perspectives from identitybased views of the firm. European Journal of Marketing, 45(9/10), 1329-1352. https://doi. org/10.1108/03090561111151781

Balmer, J. M. T., Abratt, R., \& Kleyn, N. (2016). Corporate brands and corporate marketing: Emerging trends in the big five eco-system. Journal of Brand Management, 23(1), 3-7. https://doi.org/10.1057/bm.2015.51

Balmer, J. M. T., \& Chen, W. (2016). Corporate heritage tourism brand attractiveness and national identity. Journal of Product and Brand Management, 25(3), 223-238. https://doi.org/10.1108/JPBM-08-2015-0959

Balmer, J. M. T., \& Gray, E. R. (2003). Corporate brands: what are they? What of them? European Journal of Marketing, 37(7/8), 972-997. https://doi.org/10.1108/03090560310477627

Balmer, J. M. T., \& Greyser, S. A. (2006). Corporate marketing: Integrating corporate identity, corporate branding, corporate communications, corporate image and corporate reputation. European Journal of Marketing, 40(7/8), 730-741. https:// doi.org/10.1108/03090560610669964

Balmer, J. M. T., Powell, S. M., \& Greyser, S. A. (2011). Explicating Ethical Corporate Marketing. Insights from the BP Deepwater Horizon Catastrophe: The Ethical Brand that Exploded and then Imploded. Journal of Business Ethics, 102(1), 1-14. https://doi.org/10.1007/s10551-011-0902-1

Balmer, J. M. T., Powell, S. M., Kernstock, J., \& Brexendorf, T. O. (2017). Advances in Corporate Branding. Advances in Corporate Branding. London. https://doi.org/10.1057/978-1-352-00008-5

Balmer, J. M. T. (2017). The corporate identity, total corporate communications, stakeholders' attributed identities, identifications and behaviours continuum. European Journal of Marketing, 51(9/10), 1472-1502. https://doi.org/10.1108/ EJM-07-2017-0448

Balmer, J. M. T., \& Wang, W. Y. (2016a). The corporate brand and strategic direction: Senior business school managers' cognitions of corporate brand building and management. Journal of Brand Management, 23(1), 8-21. https://doi. org/10.1057/bm.2015.45

Balmer, J. M. T., \& Wang, W. Y. (2016b). Why Business School Managers are a Key Corporate Brand Stakeholder Group. International Studies of Management and Organization, 46(4), 247-255. https://doi.org/10.1080/00208825.2016.1140521

Bawa, A., \& Saha, A. (2016). Strength of corporate social responsibility as a corporate brand association: general public perspective. Decision, 43(4), 313-332. https://doi.org/10.1007/s40622-016-0125-5 
Biraghi, S., \& Gambetti, R. C. (2015). Corporate branding: Where are we? A systematic communication-based inquiry. Journal of Marketing Communications, 21(4), 260-283. https://doi.org/10.1080/13527266.2013.768535

Brexendorf, T. O., \& Keller, K. L. (2017). Leveraging the corporate brand: The importance of corporate brand innovativeness and brand architecture. European Journal of Marketing, 51(9/10), 1530-1551. https://doi.org/10.1108/EJM-07-2017-0445

Brown, T. J., Dacin, P. A., Pratt, M. G., \&Whetten, D. A. (2006). Identity, intended image, construed image, and reputation: An interdisciplinary framework and suggested terminology. Journal of the Academy of Marketing Science, 34(2), 99-106. https://doi.org/10.1177/0092070305284969

Buil, I., Catalán, S., \& Martínez, E. (2016). The importance of corporate brand identity in business management: An application to the UK banking sector. BRQ Business Research Quarterly, 19(1), 3-12. https://doi.org/10.1016/j.brq.2014.11.001

Capriotti, P. (2013). Planificacion estrategica de la imagencorporativa. (IIRP - Instituto de Investigación en Relaciones Públicas, Ed.) (4ta ed.). Malaga. Retrieved from http://www.bidireccional.net

Codina, L. (2018). Revisiones sistematizadas para trabajos académicos: Conceptos, fases y bibliografía. Retrieved from https://www.lluiscodina.com/revisiones-sistematizadas-fundamentos/

Cornelissen, J. (2008). Corporate Communication: A Guide to Theory and Practice. (2nd ed.). Los Angeles: SAGE.

Costa, J. (1994). Imagen global : evolución del diseño de identidad. CEAC.

Costa, J. (2001). Imágen corporativa en el siglo XXI. La Crujía.

Daffey, A., \& Abratt, R. (2002). Corporate branding in a banking environment. Corporate Communications: An International Journal, 7(2), 87-91. https://doi.org/10.1108/13563280210426142

Dall'Olmo Riley, F., \& de Chernatony, L. (2000). The Service Brand as Relationships Builder. British Journal of Management, 11(2), 137-150. https://doi.org/10.1111/1467-8551.t01-1-00156

Dunnion, B., \& Knox, S. (2004). Understanding And Managing Corporate Brands: A System Dynamics Perspective. Retrieved from https://core.ac.uk/download/pdf/140266.pdf

Einwiller, S., \& Will, M. (2002). Towards an integrated approach to corporate branding - an empirical study. Corporate Communications: An International Journal, 7(2), 100-109. https://doi.org/10.1108/13563280210426160

Ferilli, G., Sacco, P. L., Teti, E., \& Buscema, M. (2016). Top corporate brands and the global structure of country brand positioning: An AutoCM ANN approach. Expert Systems with Applications, 66, 62-75. https://doi.org/10.1016/j.eswa.2016.08.054 Fetscherin, M., \& Usunier, J. (2012). Corporate branding: an interdisciplinary literature review. European Journal of Marketing, 46(5), 733-753. https://doi.org/10.1108/03090561211212494

González-Oñate, C. (2008). Cuatro: la nueva estrategia de marca televisiva para conectar con los jóvenes. Comunicar, XVI, 357-366. https://doi.org/10.3916/c31-2008-03-018

Harris, F., \& de Chernatony, L. (2001). Corporate branding and corporate brand performance. European Journal of Marketing, 35(3/4), 441-456. https://doi.org/10.1108/03090560110382101 
He, H.-W., \& Balmer, J. M. T. (2006). Alliance brands: Building corporate brands through strategic alliances?, 13(4), 16.

Heller, M. (2016). "Outposts of Britain” the General Post Office and the birth of a corporate iconic brand, 1930-1939. European Journal of Marketing, 50(3-4), 358-376. https://doi.org/10.1108/EJM-11-2014-0691

Iglesias, O., \& Saleem, F. Z. (2015). How to support consumer-brand relationships: The role of corporate culture and human resource policies and practices. Marketing Intelligence \& Planning, 33(2), 216-234. https://doi.org/10.1108/ MIP-10-2014-0196

Ind, N. (1997). The corporate brand. New York University Press.

Jo Hatch, M. J., \& Schultz, M. (2001). Are the strategic stars aligned for your corporate brand? Harvard Business Review, $79(2)$.

Jo Hatch, M., \& Schultz, M. (2003a). Bringing the corporation into corporate branding. European Journal of Marketing, 37(7/8), 1042-1064. https://doi.org/10.1108/03090560310477654

Jo Hatch, M., \& Schultz, M. (2003b). Bringing the corporation into corporate branding. European Journal of Marketing. https://doi.org/10.1108/03090560310477654

Kay, M. J. (2006). Strong brands and corporate brands. European Journal of Marketing, 40(7/8), 742-760.

Keller, K. L., \& Aaker, D. A. (1998). The Impact of Corporate Marketing on a Company's Brand Extensions. Corporate Reputation Review, 1(4), 356-378. https://doi.org/10.1057/palgrave.crr.1540057

King, S. (1991). Brand-building in the 1990s. Journal of Marketing, 7, 3-13. Retrieved from https://content.ebscohost. com/ContentServer.asp?T=P\&P=AN\&K=17429668\&S=R\&D=bsu\&EbscoContent=dGJyMMTo50Sep7A4v\%2BbwOLCmr1 GeprFSsaq4S7eWxWXS\&ContentCustomer=dGJyMPGpskulp69PuePfgeyx43zx

Knox, S., \& Bickerton, D. (2003). The six conventions of corporate branding. European Journal of Marketing, 37(7/8), 9981016. https://doi.org/10.1108/03090560310477636

Koporcic, N., \& Halinen, A. (2018). Interactive Network Branding. IMP Journal, 12(2), 392-408. https://doi.org/10.1108/ IMP-05-2017-0026

Machi, L. y McEvoy, B. (2009) The literature review: six steps to success. Thousand Oaks, Ca: Sage.

Malmelin, N., \& Moisander, J. (2014). Brands and Branding in Media Management-Toward a Research Agenda. JMM International Journal on Media Management. https://doi.org/10.1080/14241277.2014.898149

Markovic Markovic, S., Iglesias Bedós, O., Singh, J., \& Sierra Olivera, V. (2018). How does the perceived ethicality of corporate service brands influence loyalty and positive word-of-mouth? Analyzing the roles of empathy, affective commitment, and perceived quality - ESADE Knowledge. Journal of Business Ethics, 148(4), 721-740. Retrieved from http://www.esadeknowledge.com/view/how-does-the-perceived-ethicality-of-corporate-service-brands-influence-loyalty-and-positive-word-of-mouth-analyzing-the-roles-of-empathy-affective-commitment-and-perceived-quality-187998 
Melewar, T. ., \& Jenkins, E. (2002). Defining the Corporate Identity Construct. Corporate reputation review, 5(11), 76-91. Retrieved from https:// content.ebscohost.com/ContentServer.asp?T=P\&P=AN\&K=6876701\&S=R\&D=bsu\&EbscoConten$\mathrm{t}=\mathrm{dGJyMNLr40SeqLU4v \% 2BvlOLCmr1GeprRSr624SLSWxWXS \& ContentCustomer=dGJyMPGpskulp69PuePfgeyx43zx}$

Merrilees, B. (2017). Experience-centric branding: Challenges and advancing a new mantra for corporate brand governance. Journal of Brand Management, 24(1), 1-13. https://doi.org/10.1057/s41262-017-0027-7

Mingione, M. (2015). "Inquiry into corporate brand alignment: a dialectical analysis and directions for future research". Journal of Product \& Brand Management, Vol. 24 Issue: 5, 518-536, https://doi.org/10.1108/JPBM-05-2014-0617

Mohan, M., Voss, K. E., Jiménez, F. R., \& Gammoh, B. S. (2018). Corporate brands as brand allies. Journal of Product and Brand Management, 27(1), 41-56. https://doi.org/10.1108/JPBM-01-2016-1080

Monfort de Bedoya, A., \& Villagra García, N. (2014). Claves para entender la marca corporativa. Fe d'erratas.

Podnar, K. (2015). Corporate Communication: A marketing viewpoint. New York, NY: Routledge, Taylor \& Francis Group

Santos, F. P., Burghausen, M., \& Balmer, J. M. T. (2016). Heritage branding orientation: The case of Ach. Brito and the dynamics between corporate and product heritage brands. Journal of Brand Management, 23(1), 67-88. https://doi. org/10.1057/bm.2015.48

Schmidt, H. J., \& Redler, J. (2018). How diverse is corporate brand management research? Comparing schools of corporate brand management with approaches to corporate strategy. Journal of Product \& Brand Management, 27(2), 185-202. https://doi.org/10.1108/JPBM-05-2017-1473

Schroeder, J. E. (2017). Corporate branding in perspective: a typology. European Journal of Marketing, 51(9-10), 15221529. https://doi.org/10.1108/EJM-07-2017-0450

Schultz, M., Antorini, Y. M., \& Csaba, F. F. (2005). Corporate branding : purposelpeople/process : towards the second wave of corporate branding. Copenhagen Business School Press.

Schultz, M., \& de Chernatony, L. (2002). Introduction. The Challenges of Corporate Branding. Corporate Repputation Review, 5(2/3), 105-112. Retrieved from https://content.ebscohost.com/ContentServer.asp?T=P\&P=AN\&K=7539206\&S=R\&$\mathrm{D}=$ bsu\&EbscoContent=dGJyMMvl7ESep7M4yOvqOLCmr1GeprZSsae4SK\%2BWxWXS\&ContentCustomer=dGJyMPGpskulp69PuePfgeyx43zx

Schwaiger, M., \& Sarstedt, M. (2011). Corporate branding in a turbulent environment. Journal of Brand Management, 19(3), 179-181. https://doi.org/10.1057/bm.2011.35

Sevel, L., Abratt, R., \& Kleyn, N. (2018). Managing across a corporate and product brand portfolio: evidence from a large South African service organization. Journal of Product and Brand Management, 27(1), 18-28. https://doi.org/10.1108/ JPBM-05-2016-1182

Shahri, M. H. (2011). The effectiveness of corporate branding strategy in multi business companies. Australian Journal of Business and Management Research, 1(16), 51-59. 
Sierra, V., Iglesias, O., Markovic, S., \& Singh, J. J. (2017). Does Ethical Image Build Equity in Corporate Services Brands? The Influence of Customer Perceived Ethicality on Affect, Perceived Quality, and Equity. Journal of Business Ethics, 144(3), 661-676. https://doi.org/10.1007/s10551-015-2855-2

Sørensen, A. R., Korsager, E. M., \& Heller, M. (2018). A bittersweet past: The negative equity of corporate heritage brands. Journal of Consumer Culture, O(0), 1-19. https://doi.org/10.1177/1469540518773803

Stuart, H. J. (2011). An identity-based approach to the sustainable corporate brand. Corporate Communications, 16(2), 139-149. https://doi.org/10.1108/13563281111141660

Sujchaphong, N., Nguyen, B., \& Melewar, T. C. (2017). Towards a branding oriented higher education sector: An overview of the four perspectiver on university marketing studies. The Marketing Review, 17(1), 87-116. https://doi.org/10.1362/1 46934717X14909733966128

Törmälä, M., \& Gyrd-Jones, R. I. (2017). Development of new B2B venture corporate brand identity: A narrative performance approach. Industrial Marketing Management, 65(May), 76-85. https://doi.org/10.1016/j.indmarman.2017.05.002

Uggla, H. (2006). The corporate brand association base: A conceptual model for the creation of inclusive brand architecture Article information. European Journal of Marketing, 40(7/8), 785-802. https://doi.org/10.1108/03090560610669991

Urde, M., Greyser, S. A., \& Balmer, J. M. T. (2007). Corporate brands with a heritage. Journal of Brand Management, 15(1), 4-19. https://doi.org/10.1057/palgrave.bm.2550106

Vallaster, C., Lindgreen, A., \& Maon, F. (2012). Strategically Leveraging Corporate Social Responsibility: A Corporate Branding Perspective. California Management Review, 54(3), 34-60. https://doi.org/10.1525/cmr.2012.54.3.34

van Riel, C. B. M. (1995). Principles of corporate communication. Prentice Hall.

van Riel, C. B. M., \& Fombrun, C. J. (2008). Essentials of corporate communication : implementing practices for effective reputation management. London; New York: Routledge.

Villafañe, J. (1998). Imagen positiva : gestión estratégica de la imagen de las empresas. Madrid: Pirámide.

Villafañe, J. (2012). La buena empresa: propuesta para una teoría de la reputación corporativa. Madrid: Pearson Educacion. Villafañe Justo. (1999). La Gestión profesional de la imagen corporativa. Madrid: Piramide

Villagra, N., López, B., \& Monfort, A. (2015). La gestión de intangibles y la marca corporativa: iha cambiado algo en la relación entre las empresas y la sociedad? Revista Latina de Comunicacion Social, 70, 793-812. https://doi.org/10.4185/ RLCS-2015-1072

Wiedmann, K.-P., Hennigs, N., Schmidt, S., \&Wuestefeld, T. (2011). The importance of brand heritage as a key performance driver in marketing management. Journal of Brand Management, 19(3), 182-194. https://doi.org/10.1057/bm.2011.36 Wimmer, R., \& Dominick, J. (2011). Mass media research General Mass Communication (9th ed.). Boston: Wadsworth, Cengage Learning. 


\section{Anexos}

Anexo 1. Bases de datos incluidas en la herramienta de búsqueda

\begin{tabular}{|l|c|}
\hline \multicolumn{1}{|c|}{ Base de Datos } & No Publicaciones \\
\hline Academic OneFile & 1 \\
\hline Academic Search Index & 2 \\
\hline BASE & 42 \\
\hline Business Insights Global & 6 \\
\hline Business Source Ultimate & 7 \\
\hline Complementary Index & 56 \\
\hline Directory of Open Access Journals & 6 \\
\hline EconLit & 1 \\
\hline ELibrary.RU & 1 \\
\hline Emerald Insight & 5 \\
\hline InfoTrac Informe & 1 \\
\hline PsyclNFO & 8 \\
\hline RCAAP & 3 \\
\hline SciELO & 1 \\
\hline ScienceDirect & 4 \\
\hline Scopus & 59 \\
\hline Social Sciences Citation Index & 9 \\
\hline Supplemental Index & 5 \\
\hline RePEc & 1 \\
\hline \hline Total & 218 \\
\hline
\end{tabular}

Fuente: Elaboración propia a partir de información sobre UNIKA 
Anexo 2. Revistas 2016-2018

\begin{tabular}{|c|c|c|c|}
\hline Revista 2016-2018 & Área temática & $\begin{array}{l}\mathrm{N}^{\circ} \text { artículos } \\
\text { publicados }\end{array}$ & Categoría \\
\hline Advanced Science Letters & $\begin{array}{l}\text { Computer Science/Energy/Engineering/Environmental } \\
\text { Science/Mathematics/Social Sciences }\end{array}$ & 1 & \\
\hline Asia Pacific Journal of Human Resources & Business, Management and Accounting & 1 & \\
\hline Asia Pacific Journal of Marketing and Logistics & Business, Management and Accounting & 2 & Marketing \\
\hline BRQ Business Research Quarterly & $\begin{array}{l}\text { Business, Management and Accounting/Economics, } \\
\text { Econometrics and Finance }\end{array}$ & 1 & \\
\hline Business ethics quarterly & $\begin{array}{l}\text { Arts and Humanities/ Business, Management and Accounting/ } \\
\text { Economics, Econometrics and Finance }\end{array}$ & 2 & \\
\hline Cadernos EBAPE.BR. & Management & 1 & \\
\hline Cogent Business \& Management & $\begin{array}{l}\text { Management/Marketing/Operations Research, Information } \\
\text { \& Technology/Accounting, Corporate Governance \& Business } \\
\text { Ethics/Banking \& Finance }\end{array}$ & 1 & \\
\hline Competition Forum & Business & 1 & \\
\hline Corporate Reputation Review & Business, Management and Accounting & 2 & \\
\hline $\begin{array}{l}\text { Corporate Social Responsibility and Environmental } \\
\text { Management }\end{array}$ & $\begin{array}{l}\text { Business, Management and Accounting/Environmental } \\
\text { Science/Social Sciences }\end{array}$ & 1 & \\
\hline Critical Studies in Education & Social Sciences & 1 & \\
\hline $\begin{array}{l}\text { Decision:Official Journal of the Indian Institute of } \\
\text { Management Calcutta }\end{array}$ & Management & 1 & \\
\hline Economic Annals-XXI & Economics, Econometrics and Finance/Social Sciences & 1 & \\
\hline Ekonomika Poljoprivrede & Economics & 1 & \\
\hline European Journal of Marketing & Business, Management and Accounting & 5 & Marketing \\
\hline Expert Systems with Applications & Computer Science/Engineering & 1 & \\
\hline Global Business Review & Business, Management and Accounting & 1 & \\
\hline Handel Wewnêtrzny & Economics & 1 & \\
\hline Health Marketing Quarterly & Business, Management and Accounting/Health Professions & 1 & Marketing \\
\hline Humanomics & Arts and Humanities/Economics, Econometrics and Finance & 1 & \\
\hline $\begin{array}{l}\text { IMP Journal (merge Journal of Business \& Industrial } \\
\text { Marketing) }\end{array}$ & Marketing & 1 & \\
\hline Industrial Management \& Data Systems & $\begin{array}{l}\text { Business, Management and Accounting/Computer Science/ } \\
\text { Engineering }\end{array}$ & 1 & \\
\hline Industrial marketing management & Business, Management and Accounting & 1 & Marketing \\
\hline International Journal of Bank Marketing & Business, Management and Accounting & 2 & Marketing \\
\hline International Journal of Hospitality Management & Business, Management and Accounting & 1 & \\
\hline International Journal of Innovation and Learning & Business, Management and Accounting/Social Sciences & 1 & \\
\hline $\begin{array}{l}\text { International Journal of Innovation and Technology } \\
\text { Management }\end{array}$ & Business, Management and Accounting & 1 & \\
\hline International Journal of Marketing Studies & Marketing & 1 & \\
\hline International Journal of Psychology & Arts and Humanities/Medicine/Psychology & 1 & \\
\hline $\begin{array}{l}\text { International Journal of Retail \& Distribution } \\
\text { Management }\end{array}$ & Business, Management and Accounting & 1 & Marketing \\
\hline $\begin{array}{l}\text { International Journal of Simulation: Systems, Science } \\
\text { and Technology }\end{array}$ & Computer Science/Mathematics & 1 & Marketing \\
\hline
\end{tabular}




\begin{tabular}{|c|c|c|c|}
\hline International Marketing Review & $\begin{array}{l}\text { Business, Management and Accounting/ Economics, } \\
\text { Econometrics and Finance }\end{array}$ & 2 & Marketing \\
\hline Internext: Revista Eletrônica de Negócios Internacionais & Management & 1 & \\
\hline Journal of Brand Strategy & Business, Management and Accounting & 1 & Marketing \\
\hline Journal of Business Ethics & $\begin{array}{l}\text { Arts and Humanities/Business, Management and Accounting/ } \\
\text { Economics, Econometrics and Finance/Social Sciences }\end{array}$ & 2 & \\
\hline Journal of Economics & Microeconomic & 1 & \\
\hline Journal of Fashion Marketing \& Management & Business, Management and Accounting & 1 & Marketing \\
\hline Journal of General Management & Business, Management and Accounting & 1 & \\
\hline Journal of Marketing Communications & Business, Management and Accounting & 1 & Marketing \\
\hline Journal of Strategic Marketing & Business, Management and Accounting & 3 & Marketing \\
\hline Journal of Technology Management \& Innovation & Business, Management and Accounting & 1 & \\
\hline Journal of the Academy of Marketing Science & $\begin{array}{l}\text { Business, Management and Accounting/Economics, } \\
\text { Econometrics and Finance }\end{array}$ & 1 & Marketing \\
\hline Management \& Labour Studies & Management/ Labour & 1 & \\
\hline Marketing Intelligence \& Planning & Business, Management and Accounting & 2 & Marketing \\
\hline $\begin{array}{l}\text { People \& Strategy: Journal of the human resource } \\
\text { planning society }\end{array}$ & Economy & 1 & \\
\hline Pertanika Journal of Social Sciences and Humanities & Social sciences & 1 & \\
\hline Place Branding \& Public Diplomacy & Business, Management and Accounting & 1 & Marketing \\
\hline Procedia Computer Science & Computer sciences & 1 & \\
\hline $\begin{array}{l}\text { Proceedings of the 12th Global Brand Conference of the } \\
\text { Academy of Marketing, School of Business and Economic. }\end{array}$ & Marketing & 1 & \\
\hline Scandinavian Journal of Management & Business, Management and Accounting/Psychology & 1 & \\
\hline Sebelas Maret Business Review & Business and Management & 1 & \\
\hline Turizam : meunarodni znanstveno-struni asopi & Tourism & 1 & \\
\hline Total & & 112 & \\
\hline
\end{tabular}

Fuente: elaboración propia 
Anexo 3. Áreas temáticas de revistas y cantidad de publicaciones académicas

\begin{tabular}{|c|c|c|}
\hline \multirow[t]{2}{*}{ Áreas temáticas } & \multicolumn{2}{|r|}{$\mathbf{N}^{\circ}$} \\
\hline & $\mathrm{N}^{\circ}$ de revistas & $\begin{array}{c}\text { Publicaciones } \\
\text { académicas }\end{array}$ \\
\hline Arts and Humanities (5JR) & 5 & 7 \\
\hline Banking \& Finance & 1 & 1 \\
\hline Business, Management and Accounting (SJR) & 41 & 83 \\
\hline Commerce & 1 & 1 \\
\hline Communication & 2 & 3 \\
\hline Computer Science (SJR) & 6 & 7 \\
\hline Economics, Econometrics and Finance (SJR) & 10 & 15 \\
\hline Energy (SJR) & 1 & 1 \\
\hline Engineering (5IR) & 3 & 3 \\
\hline Environmental Science (SJR) & 2 & 2 \\
\hline Health Porfession (5JR) & 1 & 1 \\
\hline Innovation & 1 & 1 \\
\hline International affairs & 1 & 1 \\
\hline Labour & 1 & 1 \\
\hline Marketing & 4 & 4 \\
\hline Mathematics (SIR) & 2 & 2 \\
\hline Medicine (5JR) & 1 & 1 \\
\hline Psychology (SJR) & 3 & 3 \\
\hline Social Sciences (5IR) & 13 & 15 \\
\hline Technological development & 1 & 1 \\
\hline Tourism & 1 & 1 \\
\hline
\end{tabular}

Fuente: Elaboración propia en base software WordCounter 